Supporting information

\title{
Reaction Mechanism of Ethanol on Model Cobalt Catalysts: DFT Calculations
}

\begin{abstract}
Meng-Ru Li ${ }^{1}$, Jun Chen ${ }^{1}$ and Gui-Chang Wang ${ }^{1,2, *}$
( ${ }^{1}$ Department of Chemistry, Key Laboratory of Advanced Energy Materials Chemistry (Ministry of Education) and Collaborative Innovation Center of Chemical Science and Engineering (Tianjin), Nankai University, Tianjin 300071, P. R. China; ${ }^{2}$ State Key Laboratory of Coal Conversion, Institute of Coal Chemistry, Chinese Academy of Sciences, Taiyuan, 030001, P. R. China)
\end{abstract}

*Corresponding author: Gui-Chang Wang. E-mail: wangguichang@ nankai.edu.cn

Telephone: +86-22-23503824(O) Fax: +86-22-23502458 


\section{Contents:}

Table S1 Effect of magnitude of (U-J) on the adsorption energies of ethanol on $\mathrm{Co}^{2+}$ and $\mathrm{Co}^{3+}$ sites

Table S2. Calculated reaction energies $(\Delta \mathrm{E})$, energy barriers $\left(\mathrm{E}_{\mathrm{a}}\right)$, and the breaking or forming bond length (d) of TSs on Co (0001) in this work

Table S3. Calculated reaction energies $(\Delta \mathrm{E})$, energy barriers $\left(\mathrm{E}_{\mathrm{a}}\right)$, and the breaking or forming bond lengths of TSs on $\mathrm{Co}_{3} \mathrm{O}_{4}(110)$-A in this work

Table S4. The analysis of reaction energies in the form of $\mathrm{CH}_{3} \mathrm{CHO}$ and $\mathrm{C}-\mathrm{C}$ cleavage on $\mathrm{Co}^{2+}$ and $\mathrm{Co}^{3+}$ sites

Table S5. Calculated reaction energies $(\Delta \mathrm{E})$, energy barriers $\left(\mathrm{E}_{\mathrm{a}}\right)$, and the breaking or forming bond lengths of TSs of C-C cleavages in Figure 4 on $\mathrm{Co}^{3+}$ and $\mathrm{Co}^{2+}$ sites

Figure S1. The PDOS of $\mathrm{Co}^{3+}$ and $\mathrm{Co}^{2+}$ sites on $\mathrm{Co}_{3} \mathrm{O}_{4}$ and $\mathrm{Co}^{0}$ site on $\mathrm{Co}(0001)$

Figure S2. Optimized configurations for the main species involved in the reaction on Co (0001)

Figure S3. Optimized configurations for the main species involved in the reaction on $\mathrm{Co}^{3+}, \mathrm{Co}^{2+}$ sites on $\mathrm{Co}_{3} \mathrm{O}_{4}(110)-\mathrm{A}$ surface

Figure S4. The energy profiles for ethanol reaction mechanism on $\mathrm{Co}^{0}$ site

Figure S5. The calculated TS structures for possible steps relevant to ethanol reaction on $\operatorname{Co}(0001)$

Figure S6. The reaction profiles and main configurations for the ethanol reaction mechanism on $\mathrm{Co}^{2+} \mathrm{site}^{2}$

Figure S7.The calculated TS structures for possible steps relevant to ethanol reaction on $\mathrm{Co}^{2+}$ site

Figure S8. The reaction profiles and main configurations for the ethanol reaction mechanism on $\mathrm{Co}^{3+}$ site

Figure S9. The calculated TS structures for possible steps relevant to ethanol reaction on $\mathrm{Co}^{3+}$ site 
Table S1. Effect of magnitude of (U-J) on the adsorption energies of ethanol on $\mathrm{Co}^{2+}$ and $\mathrm{Co}^{3+}$ sites (unit: eV)

\begin{tabular}{llllc}
\hline U-J & 1.00 & 3.00 & 3.50 & 5.00 \\
\hline $\mathrm{Co}^{2+}$ site & -0.50 & -0.48 & -1.06 & -0.40 \\
$\mathrm{Co}^{3+}$ site & -1.02 & -0.57 & -1.08 & -0.24 \\
\hline
\end{tabular}

- The set of (U-J) of $3.5 \mathrm{eV}$ was chosen for relative high adsorption energy of ethanol on $\mathrm{Co}^{2+}$ and $\mathrm{Co}^{3+} \mathrm{sites}$

Table S2. Calculated reaction energies $(\Delta \mathrm{E})$, energy barriers $\left(E_{a}\right)$, and the breaking or forming bond length $(d)$ of TSs on Co (0001) in this work ${ }^{\mathrm{a}}$

\begin{tabular}{|c|c|c|c|}
\hline Reaction & $\Delta \mathrm{E} / \mathrm{eV}$ & $\mathrm{E}_{\mathrm{a}} / \mathrm{eV}$ & $\mathrm{d} / \AA$ \\
\hline $\mathrm{M} 1 \mathrm{CH}_{3} \mathrm{CH}_{2} \mathrm{OH}+* \rightarrow \mathrm{CH}_{3} \mathrm{CH}_{2} \mathrm{OH}^{*}$ & -0.30 & - & - \\
\hline $\mathrm{M} 2 \mathrm{CH}_{3} \mathrm{CH}_{2} \mathrm{OH}^{*+} * \rightarrow \mathrm{CH}_{3} \mathrm{CH}_{2} \mathrm{O}^{*}+\mathrm{H}^{*}$ & $-0.62(-0.78)$ & $0.79(0.57)$ & 1.40 \\
\hline $\mathrm{M} 3 \mathrm{CH}_{3} \mathrm{CH}_{2} \mathrm{O}^{*+} * \mathrm{CH}_{3} \mathrm{CHO}^{*}+\mathrm{H}^{*}$ & $0.71(0.57)$ & $1.20(1.02)$ & 1.52 \\
\hline $\mathrm{M} 4 \mathrm{CH}_{3} \mathrm{CHO}^{*} \rightarrow \mathrm{CH}_{3} \mathrm{CHO}(\mathrm{g})+*$ & 0.33 & - & - \\
\hline $\mathrm{M} 5 \mathrm{CH}_{3} \mathrm{CHO}^{*+} * \mathrm{CH}_{3} \mathrm{CO} *+\mathrm{H}^{*}$ & $-0.31(-0.34)$ & $0.15(0.10)$ & 1.52 \\
\hline $\mathrm{M} 6 \mathrm{CH}_{3} \mathrm{CHO}^{*+} * \rightarrow \mathrm{CH}_{4} *+\mathrm{CO}^{*}$ & $-0.68(-0.70)$ & $0.96(0.84)$ & 2.71 \\
\hline $\mathrm{M} 7 \mathrm{CH}_{3} \mathrm{CO}^{*+} * \rightarrow \mathrm{CH}_{3} *+\mathrm{CO}^{*}$ & $-0.60(-0.72)$ & $1.02(1.01)$ & 1.86 \\
\hline $\left.\mathrm{M} 8 \mathrm{CO}^{*} \rightarrow \mathrm{CO}(\mathrm{g})\right)^{*}$ & 1.64 & - & - \\
\hline M9 $\mathrm{CH}_{3}{ }^{*}+\mathrm{H}^{*} \rightarrow \mathrm{CH}_{4}{ }^{*}+*$ & $0.06(-0.06)$ & $0.99(0.99)$ & 1.54 \\
\hline $\mathrm{M} 10 \mathrm{CH}_{4} * \rightarrow \mathrm{CH}_{4}(\mathrm{~g})+*$ & -0.10 & 0.00 & - \\
\hline $\mathrm{M} 11 \mathrm{CH}_{3} *+* \rightarrow \mathrm{CH}_{2}{ }^{*}+\mathrm{H}^{*}$ & $0.31(0.22)$ & $0.77(0.64)$ & 1.64 \\
\hline $\mathrm{M}_{12} \mathrm{CH}_{2}{ }^{*+} \rightarrow \mathrm{CH}^{*+} \mathrm{H}^{*}$ & $-0.15(-0.21)$ & $0.29(0.20)$ & 1.65 \\
\hline $\mathrm{M} 13 \mathrm{CH}^{*+} * \rightarrow \mathrm{C}^{*+} \mathrm{H}^{*}$ & $0.34(0.30)$ & $1.28(1.19)$ & 1.46 \\
\hline $\mathrm{M} 14 \mathrm{C}^{*+} \mathrm{O}^{*} \rightarrow \mathrm{CO}^{*+*}$ & $-1.07(-1.12)$ & $1.31(1.31)$ & 1.78 \\
\hline $\mathrm{M} 15 \mathrm{CO}^{*} \rightarrow \mathrm{CO}(\mathrm{g})+*$ & 1.64 & - & - \\
\hline $\mathrm{M} 16 \mathrm{H}^{*}+\mathrm{H}^{*} \rightarrow \mathrm{H}_{2}^{*}+*$ & $0.71(0.69)$ & $1.36(1.31)$ & 0.88 \\
\hline M17 $\mathrm{H}_{2}{ }^{*} \rightarrow \mathrm{H}_{2}(\mathrm{~g})+*$ & 0.02 & - & - \\
\hline
\end{tabular}

Note: ${ }^{a}$ Entries in parentheses are the ZPE-corrected values. 
Table S3. Calculated reaction energies $(\Delta \mathrm{E})$, energy barriers $\left(\mathrm{E}_{\mathrm{a}}\right)$, and the breaking or forming bond lengths of TSs on $\mathrm{Co}_{3} \mathrm{O}_{4}(110)-\mathrm{A}$ in this work ${ }^{\mathrm{a}}$

\begin{tabular}{|c|c|c|c|c|c|}
\hline Reaction $\left(\mathrm{Co}^{3+}\right.$ site $)$ & & $\Delta \mathrm{E} / \mathrm{eV}$ & & $\mathrm{E}_{\mathrm{a}} / \mathrm{eV}$ & $\mathrm{d} / \AA$ \\
\hline $\mathrm{M} 1 \mathrm{CH}_{3} \mathrm{CH}_{2} \mathrm{OH}(\mathrm{g})+* \rightarrow \mathrm{CH}_{3} \mathrm{CH}_{2} \mathrm{OH}^{*}$ & & -1.06 & & - & - \\
\hline $\mathrm{M} 2 \mathrm{CH}_{3} \mathrm{CH}_{2} \mathrm{OH}^{*}+\mathrm{O}_{3 \mathrm{f}} * \rightarrow \mathrm{CH}_{3} \mathrm{CH}_{2} \mathrm{O}^{*}+\mathrm{HO}_{3 \mathrm{f}} *$ & & $-0.86(-0.89)$ & & $0.18(0.16)$ & 1.16 \\
\hline $\mathrm{M} 3 \mathrm{CH}_{3} \mathrm{CH}_{2} \mathrm{O}^{*}+\mathrm{O}_{3 \mathrm{f}} * \rightarrow \mathrm{CH}_{3} \mathrm{CHO}^{*}+\mathrm{HO}_{3 \mathrm{f}} *$ & & $0.68(0.67)$ & & $1.13(0.93)$ & 1.36 \\
\hline $\mathrm{M} 4 \mathrm{CH}_{3} \mathrm{CHO}^{*} \rightarrow \mathrm{CH}_{3} \mathrm{CHO}(\mathrm{g})+*$ & & 0.69 & & - & - \\
\hline $\mathrm{M} 5 \mathrm{CH}_{3} \mathrm{CHO}^{*}+\mathrm{O}_{3 \mathrm{f}}{ }^{*} \rightarrow \mathrm{CH}_{2} \mathrm{CHO} *+\mathrm{HO}_{3 \mathrm{f}} *$ & & $-0.24(-0.20)$ & & $0.26(0.14)$ & 1.34 \\
\hline $\mathrm{M} 6 \mathrm{CH}_{2} \mathrm{CHO}^{*}+\mathrm{O}_{3 \mathrm{f}} * \rightarrow \mathrm{CH}_{2} \mathrm{CO}^{*}+\mathrm{HO}_{3 \mathrm{f}} *$ & & $0.05(0.00)$ & & $0.77(0.55)$ & 1.44 \\
\hline $\mathrm{M} 7 \mathrm{CH}_{2} \mathrm{CO}^{*}+\mathrm{HO}_{3 \mathrm{f}} * \rightarrow \mathrm{CHCO}^{*}+\mathrm{H}_{2}\left(\mathrm{O}_{3 \mathrm{f}}\right)^{*}$ & & $0.47(0.47)$ & & $0.91(0.78)$ & 1.34 \\
\hline $\mathrm{M} 8 \mathrm{H}_{2}\left(\mathrm{O}_{3 \mathrm{f}}\right) * \rightarrow \mathrm{H}_{2} \mathrm{O}(\mathrm{g})+\mathrm{O}_{3 \mathrm{v}} *$ & & 0.46 & & - & - \\
\hline $\mathrm{M} 9 \mathrm{CHCO}^{*}+\mathrm{O}_{3 \mathrm{f}} \mathrm{H}^{*} \rightarrow \mathrm{CCO}^{*}+\mathrm{H}_{2}\left(\mathrm{O}_{3 \mathrm{f}}\right) *$ & & $-0.06(-0.03)$ & & $0.13(0.05)$ & 1.24 \\
\hline $\mathrm{M} 10 \mathrm{H}_{2}\left(\mathrm{O}_{3 \mathrm{f}}\right) * \rightarrow \mathrm{H}_{2} \mathrm{O}(\mathrm{g})+\mathrm{O}_{3 \mathrm{v}} *$ & & 0.88 & & - & - \\
\hline $\mathrm{M}_{11} \mathrm{O}_{2}(\mathrm{~g})+\mathrm{O}_{3 \mathrm{v}}{ }^{*}+\mathrm{CCO}^{*} \rightarrow \mathrm{COCO}^{*}+\mathrm{O}_{3 \mathrm{f}}{ }^{*}$ & & -4.16 & & 0.00 & - \\
\hline $\mathrm{M} 12 \mathrm{COCO}^{*+} * \rightarrow \mathrm{CO}(1)^{*+} \mathrm{CO}(2)^{*}$ & & $-1.53(-1.55)$ & & $0.15(0.09)$ & 1.71 \\
\hline $\mathrm{M} 13 \mathrm{CO}(1)^{*} \rightarrow \mathrm{CO}(\mathrm{g})+*$ & & 0.40 & & - & - \\
\hline $\mathrm{M} 14 \mathrm{CO}(2)^{*} \rightarrow \mathrm{CO}(\mathrm{g})^{+*}$ & & 1.34 & & - & - \\
\hline $\mathrm{M} 15 \mathrm{O}_{2}(\mathrm{~g})+\mathrm{O}_{3 \mathrm{v}} *+\mathrm{CO}(1)^{*} \rightarrow \mathrm{CO}_{2}(1)^{*}+\mathrm{O}_{3 \mathrm{f}} *$ & & -5.74 & & 0.00 & - \\
\hline $\mathrm{M} 16 \mathrm{CO}_{2}(1)^{*} \rightarrow \mathrm{CO}_{2}(\mathrm{~g})+*$ & & -0.14 & & 0.00 & - \\
\hline $\mathrm{M} 172 \mathrm{HO}_{3 \mathrm{f}} * \rightarrow \mathrm{H}_{2} \mathrm{O}_{3 \mathrm{f}} *+\mathrm{O}_{3 \mathrm{f}} *$ & & $-0.20(-0.13)$ & & $0.12(0.09)$ & 1.36 \\
\hline $\mathrm{M} 18 \mathrm{H}_{2}\left(\mathrm{O}_{3 \mathrm{f}}\right) * \rightarrow \mathrm{H}_{2} \mathrm{O}(\mathrm{g})+\mathrm{O}_{3 \mathrm{v}} *$ & & 1.04 & & 0.00 & - \\
\hline $\mathrm{M} 19 \mathrm{O}_{2}(\mathrm{~g})+\mathrm{O}_{3 v}{ }^{*}+\mathrm{CO}(2)^{*} \rightarrow \mathrm{CO}_{2}(2)^{*}+\mathrm{O}_{3 \mathrm{f}}{ }^{*}$ & & -5.38 & & 0.00 & - \\
\hline $\mathrm{M} 20 \mathrm{CO}_{2}(2) * \rightarrow \mathrm{CO}_{2}(\mathrm{~g})+*$ & & 0.10 & & 0.00 & - \\
\hline Side reaction $\left(\mathrm{Co}^{3+}\right.$ site $)$ & $\Delta \mathrm{E} / \mathrm{eV}$ & & $\mathrm{E}_{\mathrm{a}} / \mathrm{eV}$ & & $\mathrm{d} / \AA$ \\
\hline 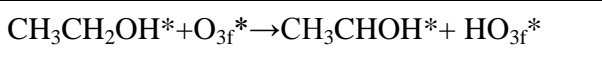 & -0.72 & & 1.36 & & 1.41 \\
\hline $\mathrm{CH}_{3} \mathrm{CH}_{2} \mathrm{OH}^{*}+\mathrm{O}_{3 \mathrm{f}}{ }^{*} \rightarrow \mathrm{CH}_{2} \mathrm{CH}_{2} \mathrm{OH}^{*}+\mathrm{HO}_{3 \mathrm{f}} *$ & 1.33 & & 1.41 & & 1.46 \\
\hline $\mathrm{CH}_{3} \mathrm{CH}_{2} \mathrm{O}^{*}+\mathrm{HO}_{3 \mathrm{f}}{ }^{*} \rightarrow \mathrm{CH}_{3} \mathrm{CHO}^{*}+\mathrm{H}_{2}\left(\mathrm{O}_{3 \mathrm{f}}\right)^{*}$ & 1.41 & & 1.96 & & 1.42 \\
\hline $\mathrm{CH}_{3} \mathrm{CH}_{2} \mathrm{O}^{*}+\mathrm{O}_{3 \mathrm{f}}{ }^{*} \rightarrow \mathrm{CH}_{2} \mathrm{CH}_{2} \mathrm{O}^{*}+\mathrm{O}_{3 \mathrm{f}} \mathrm{H}^{*}$ & 1.08 & & 1.73 & & 1.44 \\
\hline $\mathrm{CH}_{3} \mathrm{CH}_{2} \mathrm{O}^{*}+\mathrm{HO}_{3 \mathrm{f}} * \rightarrow \mathrm{CH}_{2} \mathrm{CH}_{2} \mathrm{O}^{*}+\mathrm{H}_{2}\left(\mathrm{O}_{3 \mathrm{f}}\right)^{*}$ & 2.09 & & 2.17 & & 2.29 \\
\hline $\mathrm{CH}_{3} \mathrm{CHO}^{*}+\mathrm{O}_{3 \mathrm{f}} * \rightarrow \mathrm{CH}_{3} \mathrm{CO}^{*}+\mathrm{HO}_{3 \mathrm{f}} *$ & -0.08 & & 0.42 & & 1.48 \\
\hline $\mathrm{CH}_{3} \mathrm{CHO}^{*}+\mathrm{HO}_{3 \mathrm{f}}{ }^{*} \rightarrow \mathrm{CH}_{3} \mathrm{CO}^{*}+\mathrm{H}_{2}\left(\mathrm{O}_{3 \mathrm{f}}\right)^{*}$ & 0.95 & & 1.24 & & 1.57 \\
\hline
\end{tabular}




\begin{tabular}{|c|c|c|c|c|}
\hline $\mathrm{CH}_{3} \mathrm{CHO}^{*}+\mathrm{HO}_{3 \mathrm{f}}{ }^{*} \rightarrow \mathrm{CH}_{2} \mathrm{CHO}^{*}+\mathrm{H}_{2}\left(\mathrm{O}_{3 \mathrm{f}}\right)^{*}$ & 0.54 & 1.29 & & 1.77 \\
\hline $\mathrm{CH}_{3} \mathrm{CO}^{*}+\mathrm{O}_{3 \mathrm{f}} * \rightarrow \mathrm{CH}_{2} \mathrm{CO}^{*}+\mathrm{HO}_{3 \mathrm{f}} *$ & 0.89 & 1.72 & & 1.49 \\
\hline $\mathrm{CH}_{3} \mathrm{CO}^{*}+\mathrm{HO}_{3 \mathrm{f}}{ }^{*} \rightarrow \mathrm{CH}_{2} \mathrm{CO}^{*}+\mathrm{H}_{2}\left(\mathrm{O}_{3 \mathrm{f}}\right) *$ & 0.30 & 0.46 & & 1.46 \\
\hline $\mathrm{CH}_{3} \mathrm{CO}^{*+} * \rightarrow \mathrm{CH}_{3}{ }^{*}+\mathrm{CO}^{*}$ & 1.33 & 1.52 & & 2.03 \\
\hline $\mathrm{CH}_{2} \mathrm{CO}^{*}+\mathrm{HO}_{3 \mathrm{f}}{ }^{*} \rightarrow \mathrm{CHCO}^{*}+\mathrm{H}_{2}\left(\mathrm{O}_{3 \mathrm{f}}\right) *$ & 0.97 & 1.97 & & 1.34 \\
\hline $\mathrm{CH}_{2} \mathrm{CO}^{*+} * \rightarrow \mathrm{CH}_{2} *+\mathrm{CO}^{*}$ & 0.91 & 1.04 & & 1.92 \\
\hline $\mathrm{CH}_{2} \mathrm{CHO} *+\mathrm{HO}_{3 \mathrm{f}} * \rightarrow \mathrm{CH}_{2} \mathrm{CO}^{*}+\mathrm{H}_{2}\left(\mathrm{O}_{3 \mathrm{f}}\right)^{*}$ & 1.51 & 1.80 & & 1.43 \\
\hline $\mathrm{CH}_{2} \mathrm{CHO}^{*}+\mathrm{O}_{3 \mathrm{f}}{ }^{*} \rightarrow \mathrm{CHCHO}^{*}+\mathrm{HO}_{3 \mathrm{f}} *$ & 1.06 & 2.81 & & 2.16 \\
\hline $\mathrm{CH}_{2} \mathrm{CHO}^{*}+\mathrm{HO}_{3 \mathrm{f}}{ }^{*} \rightarrow \mathrm{CHCHO}^{*}+\mathrm{H}_{2}\left(\mathrm{O}_{3 \mathrm{ff}}\right)^{*}$ & 0.43 & 0.50 & & 1.43 \\
\hline $\mathrm{CHCHO}^{*}+\mathrm{O}_{3 \mathrm{f}} * \rightarrow \mathrm{CHCO}^{*}+\mathrm{HO}_{3 \mathrm{f}} *$ & -0.25 & 1.61 & & 1.77 \\
\hline $\mathrm{CHCHO}^{*}+\mathrm{HO}_{3 \mathrm{f}} * \rightarrow \mathrm{CHCO}^{*}+\mathrm{H}_{2}\left(\mathrm{O}_{3 \mathrm{f}}\right) *$ & 1.53 & 2.94 & & 1.73 \\
\hline $\mathrm{CHCHO}^{*}+\mathrm{O}_{3 \mathrm{f}} * \rightarrow \mathrm{CCHO}^{*}+\mathrm{HO}_{3 \mathrm{f}} *$ & 0.13 & 2.15 & & 1.36 \\
\hline $\mathrm{CHCHO}^{*}+\mathrm{HO}_{3 \mathrm{f}} * \rightarrow \mathrm{CCHO}^{*}+\mathrm{H}_{2}\left(\mathrm{O}_{3 \mathrm{f}}\right) *$ & 0.77 & 0.79 & & 1.37 \\
\hline $\mathrm{CCHO}^{*}+\mathrm{O}_{3 \mathrm{f}} * \rightarrow \mathrm{CCO}^{*}+\mathrm{HO}_{3 \mathrm{f}} *$ & 1.30 & 1.45 & & 1.58 \\
\hline $\mathrm{CH}_{2} \mathrm{CO}^{*+} * \rightarrow \mathrm{CH}_{2}{ }^{*}+\mathrm{CO}^{*}$ & 1.43 & 1.95 & & 2.26 \\
\hline $\mathrm{CHCO} *+\mathrm{HO}_{3 \mathrm{f}} * \rightarrow \mathrm{CCO}^{*}+\mathrm{H}_{2}\left(\mathrm{O}_{3 \mathrm{f}}\right) *$ & -0.06 & 0.13 & & 1.24 \\
\hline $\mathrm{CHCO}^{*}+\mathrm{O}_{3 \mathrm{v}} * \rightarrow \mathrm{CH}^{*}+\mathrm{CO}^{*}$ & 0.95 & 1.34 & & 2.24 \\
\hline $\mathrm{CCO}^{*}+\mathrm{O}_{3 v} * \rightarrow \mathrm{C}^{*}+\mathrm{CO}$ & 2.32 & 2.34 & & 2.12 \\
\hline Reaction $\left(\mathrm{Co}^{2+}\right.$ site $)$ & & $\Delta \mathrm{E} / \mathrm{eV}$ & $\mathrm{E}_{\mathrm{a}} / \mathrm{eV}$ & $\mathrm{d} / \AA$ \\
\hline $\mathrm{M} 1 \mathrm{CH}_{3} \mathrm{CH}_{2} \mathrm{OH}(\mathrm{g})+* \rightarrow \mathrm{CH}_{3} \mathrm{CH}_{2} \mathrm{OH}^{*}$ & & -1.08 & 0.0 & - \\
\hline $\mathrm{M} 2 \mathrm{CH}_{3} \mathrm{CH}_{2} \mathrm{OH}^{*}+\mathrm{O}_{3 \mathrm{f}}{ }^{*} \rightarrow \mathrm{CH}_{3} \mathrm{CH}_{2} \mathrm{O}^{*}+\mathrm{HO}_{3 \mathrm{f}} *$ & & $-0.18(-0.18)$ & $0.26(0.21)$ & 1.50 \\
\hline $\mathrm{M} 3 \mathrm{CH}_{3} \mathrm{CH}_{2} \mathrm{O}^{*}+\mathrm{O}_{3 \mathrm{f}} * \rightarrow \mathrm{CH}_{3} \mathrm{CHO}^{*}+\mathrm{HO}_{3 \mathrm{f}} *$ & & $-0.77(-0.81)$ & $0.41(0.29)$ & 1.24 \\
\hline $\mathrm{M} 4 \mathrm{CH}_{3} \mathrm{CHO}^{*} \rightarrow \mathrm{CH}_{3} \mathrm{CHO}(\mathrm{g})+*$ & & 0.50 & - & - \\
\hline $\mathrm{M} 5 \mathrm{CH}_{3} \mathrm{CHO}^{*}+\mathrm{O}_{3 \mathrm{f}}{ }^{*} \rightarrow \mathrm{CH}_{3} \mathrm{CO} *+\mathrm{HO}_{3 \mathrm{f}} *$ & & $-0.03(-0.03)$ & $0.71(0.57)$ & 1.44 \\
\hline $\mathrm{M} 6 \mathrm{CH}_{3} \mathrm{CHO}^{*}+\mathrm{O}_{3 \mathrm{f}}{ }^{*} \rightarrow \mathrm{CH}_{2} \mathrm{CHO} *+\mathrm{HO}_{3 \mathrm{f}^{*}} *$ & & $-0.44(-0.37)$ & $0.03(0.00)$ & 1.28 \\
\hline $\mathrm{M} 7 \mathrm{CH}_{3} \mathrm{CO} *+\mathrm{O}_{3 \mathrm{f}} * \rightarrow \mathrm{CH}_{2} \mathrm{CO}^{*}+\mathrm{HO}_{3 \mathrm{f}} *$ & & $0.24(0.28)$ & $0.43(0.28)$ & 1.33 \\
\hline $\mathrm{M} 8 \mathrm{CH}_{2} \mathrm{CHO} *+\mathrm{O}_{3 \mathrm{f}} * \rightarrow \mathrm{CH}_{2} \mathrm{CO} *+\mathrm{HO}_{3 \mathrm{f}} *$ & & $0.68(1.06)$ & $1.26(1.26)$ & 1.56 \\
\hline $\mathrm{M} 9 \mathrm{CH}_{2} \mathrm{CO}^{*}+\mathrm{HO}_{3 \mathrm{f}} * \rightarrow \mathrm{CHCO}^{*}+\mathrm{H}_{2}\left(\mathrm{O}_{3 \mathrm{f}}\right) *$ & & $-0.39(-0.39)$ & $0.56(0.56)$ & 1.23 \\
\hline
\end{tabular}




\begin{tabular}{|c|c|c|c|c|}
\hline $\mathrm{M} 10 \mathrm{H}_{2}\left(\mathrm{O}_{3 \mathrm{f}}\right) * \rightarrow \mathrm{H}_{2} \mathrm{O}(\mathrm{g})+\mathrm{O}_{3 \mathrm{v}} *$ & & \multicolumn{2}{|l|}{0.65} & - \\
\hline $\mathrm{M} 11 \mathrm{CHCO}^{*}+\mathrm{HO}_{3 \mathrm{f}} * \rightarrow \mathrm{CCO}^{*}+\mathrm{H}_{2}\left(\mathrm{O}_{3 \mathrm{f}}\right) *$ & & $0.58(0.58)$ & $0.85(0.85)$ & 1.75 \\
\hline $\mathrm{M} 12 \mathrm{H}_{2}\left(\mathrm{O}_{3 \mathrm{f}}\right) * \rightarrow \mathrm{H}_{2} \mathrm{O}(\mathrm{g})+\mathrm{O}_{3 \mathrm{v}} *$ & & 0.84 & - & - \\
\hline $\mathrm{M}_{13} \mathrm{O}_{2}(\mathrm{~g})+\mathrm{O}_{3 \mathrm{v}}{ }^{*}+\mathrm{CCO}^{*} \rightarrow \mathrm{COCO}^{*}+\mathrm{O}_{3 \mathrm{f}} *$ & & -5.05 & - & - \\
\hline $\mathrm{M} 14 \mathrm{COCO}^{*+} * \rightarrow \mathrm{CO}(1)^{*+} \mathrm{CO}(2)^{*}$ & & $-0.36(-0.20)$ & $0.98(0.95)$ & 1.93 \\
\hline $\mathrm{M} 15 \mathrm{CO}(1)^{*} \rightarrow \mathrm{CO}(\mathrm{g})+*$ & & 0.73 & - & - \\
\hline $\mathrm{M} 16 \mathrm{CO}(2)^{*} \rightarrow \mathrm{CO}(\mathrm{g})+*$ & & 0.52 & - & - \\
\hline $\mathrm{M}_{17} \mathrm{O}_{2}(\mathrm{~g})+\mathrm{O}_{3 v}{ }^{*}+\mathrm{CO}(1)^{*} \rightarrow \mathrm{CO}_{2}(1)^{*}+\mathrm{O}_{3 f^{*}}$ & & -5.99 & - & - \\
\hline $\mathrm{M} 18 \mathrm{CO}_{2}(1)^{*} \rightarrow \mathrm{CO}_{2}(\mathrm{~g})+*$ & & -0.14 & 0.00 & - \\
\hline $\mathrm{M} 192 \mathrm{HO}_{3 \mathrm{f}} * \rightarrow \mathrm{H}_{2} \mathrm{O}_{3 \mathrm{f}} *+\mathrm{O}_{3 \mathrm{f}} *$ & & $-0.23(0.04)$ & $0.63(0.56)$ & 1.43 \\
\hline $\mathrm{M} 20 \mathrm{H}_{2}\left(\mathrm{O}_{3 \mathrm{f}}\right) * \rightarrow \mathrm{H}_{2} \mathrm{O}(\mathrm{g})+\mathrm{O}_{3 \mathrm{v}} *$ & & 0.84 & - & - \\
\hline $\mathrm{M} 21 \mathrm{O}_{2}(\mathrm{~g})+\mathrm{O}_{3 \mathrm{v}} *+\mathrm{CO}(2)^{*} \rightarrow \mathrm{CO}_{2}(2)^{*}+\mathrm{O}_{3 \mathrm{f}} *$ & & -5.92 & - & - \\
\hline $\mathrm{M} 22 \mathrm{CO}_{2}(2)^{*} \rightarrow \mathrm{CO}_{2}(\mathrm{~g})+*$ & & 0.44 & - & - \\
\hline Side reaction $\left(\mathrm{Co}^{2+}\right.$ site $)$ & $\Delta \mathrm{E} / \mathrm{eV}$ & \multicolumn{2}{|c|}{$\mathrm{E}_{\mathrm{a}} / \mathrm{eV}$} & $\mathrm{d} / \AA$ \\
\hline $\mathrm{CH}_{3} \mathrm{CH}_{2} \mathrm{OH}^{*}+\mathrm{O}_{3 \mathrm{f}} * \rightarrow \mathrm{CH}_{3} \mathrm{CHOH}^{*}+\mathrm{HO}_{3 \mathrm{f}} *$ & 1.57 & \multicolumn{2}{|c|}{1.68} & 1.48 \\
\hline $\mathrm{CH}_{3} \mathrm{CH}_{2} \mathrm{OH}^{*}+\mathrm{O}_{3 \mathrm{f}} * \rightarrow \mathrm{CH}_{2} \mathrm{CH}_{2} \mathrm{OH}^{*}+\mathrm{HO}_{3 \mathrm{f}} *$ & 1.17 & \multicolumn{2}{|c|}{1.33} & 1.46 \\
\hline $\mathrm{CH}_{3} \mathrm{CH}_{2} \mathrm{O}^{*}+\mathrm{HO}_{3 \mathrm{f}} * \rightarrow \mathrm{CH}_{3} \mathrm{CHO}^{*}+\mathrm{H}_{2}\left(\mathrm{O}_{3 \mathrm{f}}\right)^{*}$ & 0.60 & \multicolumn{2}{|c|}{2.19} & 3.31 \\
\hline $\mathrm{CH}_{3} \mathrm{CH}_{2} \mathrm{O}^{*}+\mathrm{O}_{3 \mathrm{f}}{ }^{*} \rightarrow \mathrm{CH}_{2} \mathrm{CH}_{2} \mathrm{O}^{*}+\mathrm{HO}_{3 \mathrm{f}}{ }^{*}$ & 1.51 & \multicolumn{2}{|c|}{1.63} & 1.40 \\
\hline $\mathrm{CH}_{3} \mathrm{CH}_{2} \mathrm{O}^{*}+\mathrm{HO}_{3 \mathrm{f}} * \rightarrow \mathrm{CH}_{2} \mathrm{CH}_{2} \mathrm{O}^{*}+\mathrm{H}_{2}\left(\mathrm{O}_{3 \mathrm{f}}\right)^{*}$ & 2.12 & \multicolumn{2}{|c|}{2.38} & 1.67 \\
\hline $\mathrm{CH}_{3} \mathrm{CHO}^{*}+\mathrm{HO}_{3 \mathrm{f}} * \rightarrow \mathrm{CH}_{3} \mathrm{CO}^{*}+\mathrm{H}_{2}\left(\mathrm{O}_{3 \mathrm{ff}}\right)^{*}$ & 0.24 & \multicolumn{2}{|c|}{1.84} & 1.14 \\
\hline $\mathrm{CH}_{3} \mathrm{CHO}^{*}+\mathrm{HO}_{3 \mathrm{f}}{ }^{*} \rightarrow \mathrm{CH}_{2} \mathrm{CHO}^{*}+\mathrm{H}_{2}\left(\mathrm{O}_{3 \mathrm{f}}\right)^{*}$ & 0.33 & \multicolumn{2}{|c|}{2.36} & 1.11 \\
\hline $\mathrm{CH}_{3} \mathrm{CO}^{*}+\mathrm{HO}_{3 \mathrm{f}} * \rightarrow \mathrm{CH}_{2} \mathrm{CO}^{*}+\mathrm{H}_{2}\left(\mathrm{O}_{3 \mathrm{f}}\right)^{*}$ & 0.13 & \multicolumn{2}{|c|}{1.37} & 1.60 \\
\hline $\mathrm{CH}_{3} \mathrm{CO}^{*}+* \rightarrow \mathrm{CH}_{3} *+\mathrm{CO} *$ & 1.15 & \multicolumn{2}{|c|}{1.91} & 2.14 \\
\hline $\mathrm{CH}_{2} \mathrm{CHO}^{*}+\mathrm{HO}_{3 \mathrm{f}}{ }^{*} \rightarrow \mathrm{CH}_{2} \mathrm{CO}^{*}+\mathrm{H}_{2}\left(\mathrm{O}_{3 \mathrm{f}}\right) *$ & 2.24 & \multicolumn{2}{|c|}{3.82} & 2.96 \\
\hline $\mathrm{CH}_{2} \mathrm{CHO}^{*}+\mathrm{O}_{3 \mathrm{f}} * \rightarrow \mathrm{CHCHO}^{*}+\mathrm{HO}_{3 \mathrm{f}} *$ & 2.18 & \multicolumn{2}{|c|}{2.66} & 1.58 \\
\hline $\mathrm{CH}_{2} \mathrm{CHO}^{*}+\mathrm{HO}_{3 \mathrm{f}}{ }^{*} \rightarrow \mathrm{CHCHO}^{*}+\mathrm{H}_{2}\left(\mathrm{O}_{3 \mathrm{f}}\right) *$ & 0.56 & \multicolumn{2}{|c|}{0.99} & 1.41 \\
\hline $\mathrm{CHCHO}^{*}+\mathrm{O}_{3 \mathrm{f}}{ }^{*} \rightarrow \mathrm{CHCO}^{*}+\mathrm{HO}_{3 \mathrm{f}} *$ & -0.21 & \multicolumn{2}{|c|}{1.99} & 1.19 \\
\hline $\mathrm{CHCHO}^{*}+\mathrm{HO}_{3 \mathrm{f}} * \rightarrow \mathrm{CHCO}^{*}+\mathrm{H}_{2}\left(\mathrm{O}_{3 \mathrm{f}}\right) *$ & 1.22 & \multicolumn{2}{|c|}{2.95} & 1.88 \\
\hline $\mathrm{CHCHO}^{*}+\mathrm{O}_{3 \mathrm{f}}{ }^{*} \rightarrow \mathrm{CCHO}^{*}+\mathrm{HO}_{3 \mathrm{f}} *$ & 1.12 & \multicolumn{2}{|c|}{2.09} & 1.14 \\
\hline
\end{tabular}




\begin{tabular}{llll}
\hline $\mathrm{CHCHO}^{*}+\mathrm{HO}_{3 \mathrm{f}}{ }^{*} \rightarrow \mathrm{CCHO}^{*}+\mathrm{H}_{2}\left(\mathrm{O}_{3 \mathrm{f}}\right) *$ & 1.87 & 1.88 & 1.58 \\
$\mathrm{CHCO}^{*}+* \rightarrow \mathrm{CH}^{*}+\mathrm{CO}^{*}$ & 0.29 & 2.02 & 2.46 \\
$\mathrm{CCO}^{*}+\rightarrow \mathrm{C}^{*}+\mathrm{CO}^{*}$ & 1.66 & 2.28 & 2.14 \\
\hline
\end{tabular}

Note: ${ }^{\mathrm{a}}$ Entries in parentheses are the ZPE-corrected values.

Table S4. The analysis of reaction energies in the form of $\mathrm{CH}_{3} \mathrm{CHO}$ and $\mathrm{C}-\mathrm{C}$ cleavage on $\mathrm{Co}^{2+}$ and $\mathrm{Co}^{3+}$ sites

\begin{tabular}{|c|c|c|c|c|c|c|}
\hline reaction & $\begin{array}{l}E_{\text {corr }} \\
\text { (IS) }\end{array}$ & $\begin{array}{l}E_{\text {corr }} \\
\text { (FS) }\end{array}$ & $\Delta \mathrm{E}_{\text {correction }}$ & $\begin{array}{l}E_{a d s} \\
\text { (IS) }\end{array}$ & $\begin{array}{l}E_{\mathrm{ads}} \\
(\mathrm{FS})\end{array}$ & $\Delta \mathrm{E}_{\mathrm{ads}}$ \\
\hline $\mathrm{CH}_{3} \mathrm{CH}_{2} \mathrm{O}^{*}+\mathrm{O}_{3 \mathrm{f}}{ }^{*} \rightarrow \mathrm{CH}_{3} \mathrm{CHO}^{*}+\mathrm{HO}_{3 \mathrm{f}^{*}}{ }^{*}\left(\mathrm{Co}^{2+}\right)$ & 0.81 & 0.76 & -0.06 & -2.57 & -4.09 & -1.52 \\
\hline $\mathrm{CH}_{3} \mathrm{CH}_{2} \mathrm{O}^{*}+\mathrm{O}_{3 \mathrm{f}}{ }^{*} \rightarrow \mathrm{CH}_{3} \mathrm{CHO}^{*}+\mathrm{HO}_{3 \mathrm{f}^{*}}{ }^{*}\left(\mathrm{Co}^{3+}\right)$ & 0.84 & 2.46 & 1.62 & -3.30 & -5.04 & -1.74 \\
\hline $\mathrm{COCO}^{*}+* \rightarrow 2 \mathrm{CO}^{*}\left(\mathrm{Co}^{2+}\right)$ & -0.52 & 0.22 & 0.74 & -1.95 & -1.12 & 0.83 \\
\hline $\mathrm{COCO}^{*}+* \rightarrow 2 \mathrm{CO}^{*}\left(\mathrm{CO}^{3+}\right)$ & -0.99 & -0.55 & 0.44 & -0.32 & -1.20 & -0.88 \\
\hline
\end{tabular}

Table S5. Calculated reaction energies $(\Delta \mathrm{E})$, energy barriers $\left(\mathrm{E}_{\mathrm{a}}\right)$, and the breaking or forming bond lengths of TSs of $\mathrm{C}-\mathrm{C}$ cleavages in Figure 4 on $\mathrm{Co}^{3+}$ and $\mathrm{Co}^{2+}$ sites

\begin{tabular}{|c|c|c|c|c|c|c|}
\hline \multirow{2}{*}{ Elementary reaction $\left(\mathrm{Co}^{3+}\right.$ site $)$} & \multicolumn{2}{|c|}{$\Delta \mathrm{E} / \mathrm{eV}$} & \multicolumn{2}{|c|}{$\mathrm{E}_{\mathrm{a}} / \mathrm{eV}$} & \multicolumn{2}{|c|}{$\mathrm{d} / \AA$} \\
\hline & Without $\mathrm{O}_{3 \mathrm{v}}$ & With $\mathrm{O}_{3 \mathrm{v}}$ & Without $\mathrm{O}_{3 \mathrm{v}}$ & With $\mathrm{O}_{3 \mathrm{v}}$ & Without $\mathrm{O}_{3 \mathrm{v}}$ & With $\mathrm{O}_{3 \mathrm{v}}$ \\
\hline $\mathrm{CCO}^{*+*} \rightarrow \mathrm{C}^{*}+\mathrm{CO}^{*}$ & 1.05 & 2.32 & 2.26 & 2.34 & 2.37 & 2.12 \\
\hline $\mathrm{CHCO}^{*+*} \rightarrow \mathrm{CH}^{*}+\mathrm{CO}^{*}$ & 1.00 & 0.95 & 2.68 & 1.34 & 2.49 & 2.24 \\
\hline $\mathrm{CH}_{2} \mathrm{CO} *+* \rightarrow \mathrm{CH}_{2}{ }^{*}+\mathrm{CO}^{*}$ & 1.43 & 0.91 & 1.95 & 1.04 & 2.26 & 1.92 \\
\hline $\mathrm{CH}_{3} \mathrm{CO} *+* \rightarrow \mathrm{CH}_{3} *+\mathrm{CO}^{*}$ & 1.33 & 0.36 & 1.52 & 1.12 & 2.03 & 1.99 \\
\hline $\mathrm{COCO}^{*}+* 2 \mathrm{CO}^{*}$ & - & -1.53 & - & 0.15 & - & 1.71 \\
\hline \multirow{2}{*}{ Elementary reaction $\left(\mathrm{Co}^{2+}\right.$ site $)$} & \multicolumn{2}{|c|}{$\Delta \mathrm{E} / \mathrm{eV}$} & \multicolumn{2}{|c|}{$\mathrm{E}_{\mathrm{a}} / \mathrm{eV}$} & \multicolumn{2}{|c|}{$\mathrm{d} / \AA$} \\
\hline & \multicolumn{2}{|c|}{ With $\mathrm{O}_{3 \mathrm{v}}$} & \multicolumn{2}{|c|}{ With $\mathrm{O}_{3 \mathrm{v}}$} & \multicolumn{2}{|c|}{ With $\mathrm{O}_{3 \mathrm{v}}$} \\
\hline $\mathrm{CCO} *+* \rightarrow \mathrm{C}^{*}+\mathrm{CO}^{*}$ & 1.66 & & 2.28 & & 2.14 & \\
\hline $\mathrm{CHCO} *+* \rightarrow \mathrm{CH}^{*}+\mathrm{CO}^{*}$ & 0.29 & & 2.02 & & 2.46 & \\
\hline $\mathrm{CH}_{2} \mathrm{CO} *+* \rightarrow \mathrm{CH}_{2}{ }^{*}+\mathrm{CO}^{*}$ & 1.56 & & 1.62 & & 2.46 & \\
\hline $\mathrm{CH}_{3} \mathrm{CO} *+* \rightarrow \mathrm{CH}_{3}{ }^{*}+\mathrm{CO}^{*}$ & 1.15 & & 1.68 & & 2.51 & \\
\hline $\mathrm{COCO}^{*}+* \rightarrow 2 \mathrm{CO} *$ & -0.36 & & 0.98 & & 1.96 & \\
\hline
\end{tabular}



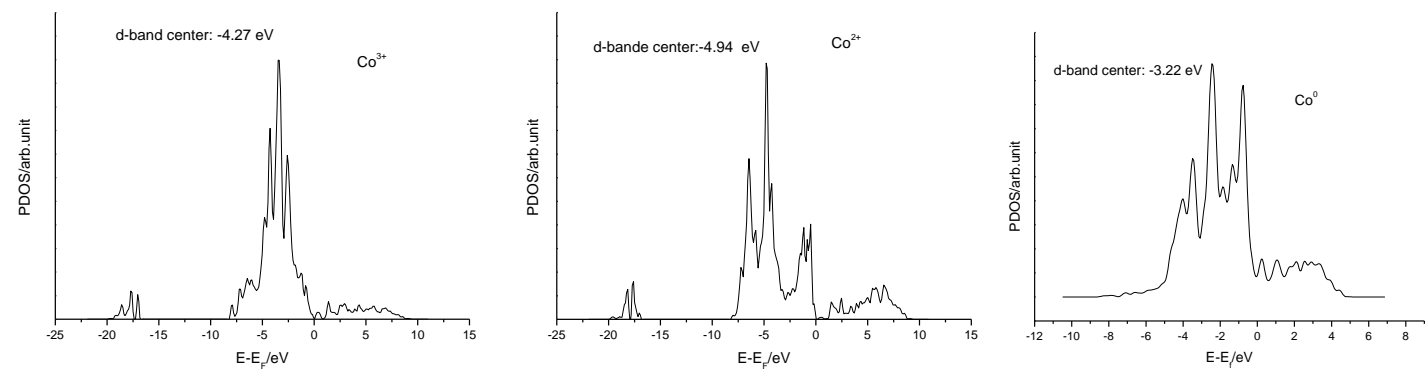

Figure S1. The PDOS of $\mathrm{Co}^{3+}$ and $\mathrm{Co}^{2+}$ sites on $\mathrm{Co}_{3} \mathrm{O}_{4}$ and $\mathrm{Co}^{0}$ site on $\mathrm{Co}(0001)$
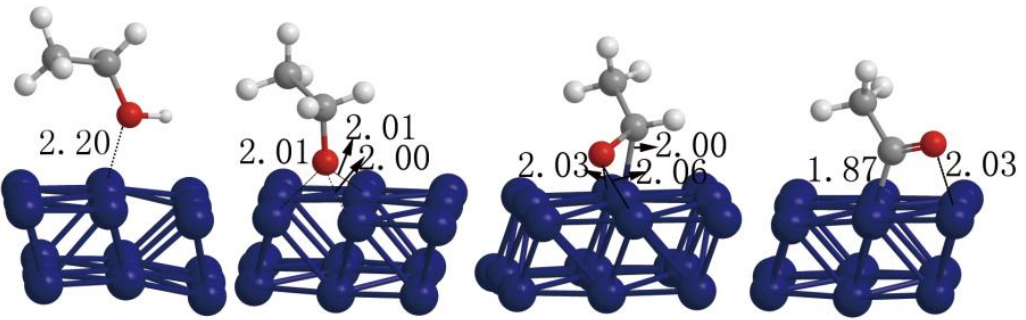

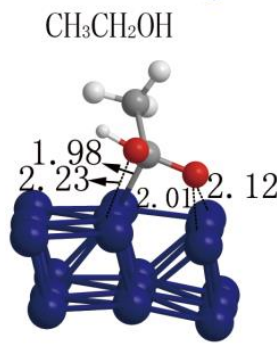

$\mathrm{CH}_{3} \mathrm{COOH}$
$\mathrm{CH}_{3} \mathrm{CH}_{2} \mathrm{O}$

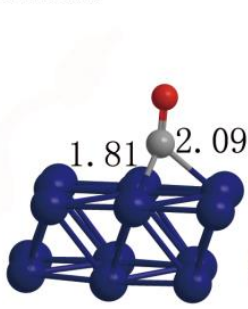

$\mathrm{CO}$

$\mathrm{CH}_{3} \mathrm{CHO}$

$\mathrm{CH}_{3} \mathrm{CO}$

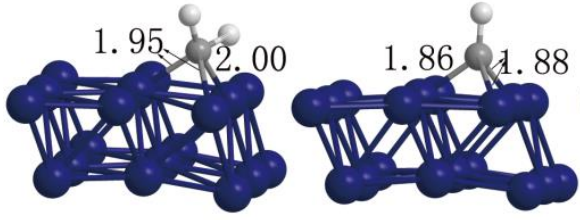

$\mathrm{CH}_{2}$
$\mathrm{CH}$

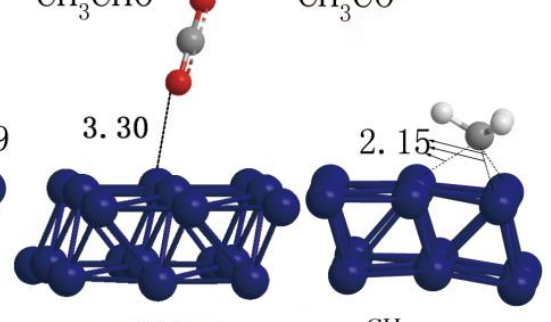

$\mathrm{CO}_{2}$

$\mathrm{CH}_{3}$

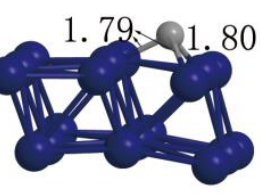

C

Figure S2. Optimized configurations for the main species involved in the reaction on Co (0001) 

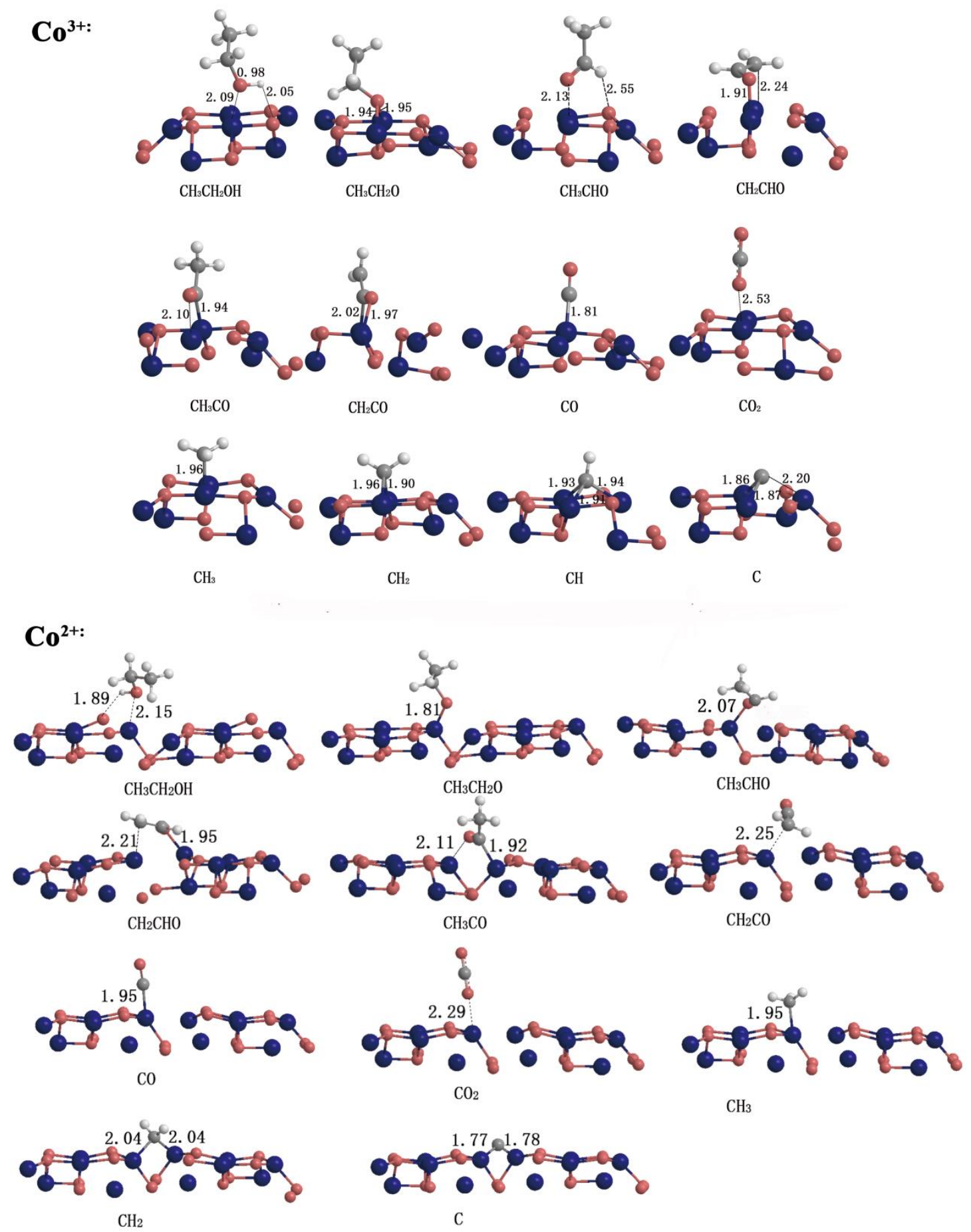

$\mathrm{CH}_{3}$

Figure S3. Optimized configurations for the main species involved in the reaction on $\mathrm{Co}^{3+}, \mathrm{Co}^{2+}$ sites on $\mathrm{Co}_{3} \mathrm{O}_{4}(110)$-A surface 


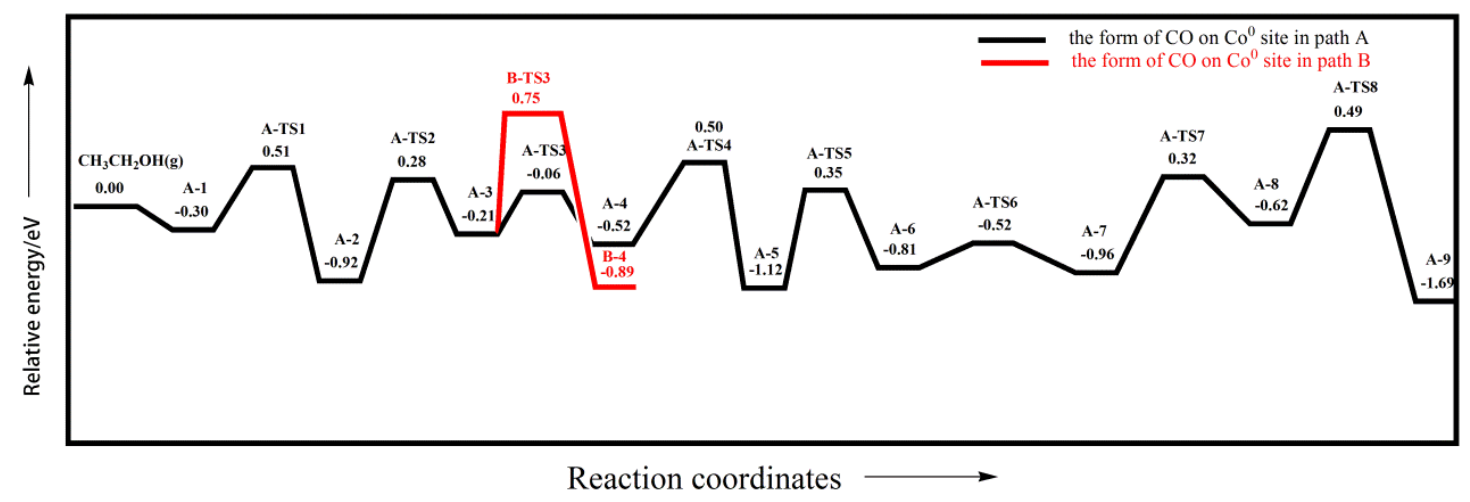

Figure S4. The energy profiles for ethanol reaction mechanism on $\mathrm{Co}^{0}$ site

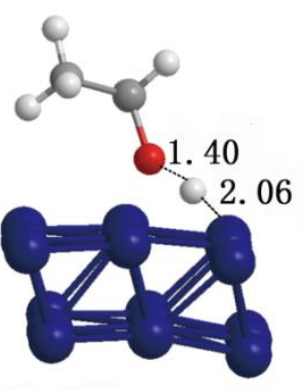

$\mathrm{CH}_{3} \mathrm{CH}_{2} \mathrm{OH}=\mathrm{CH}_{3} \mathrm{CH}_{2} \mathrm{O}+\mathrm{H}$

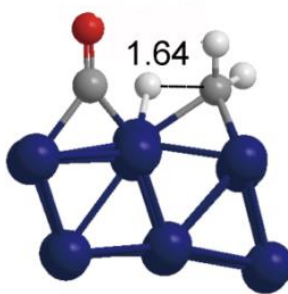

$\mathrm{CH}_{3}=\mathrm{CH}_{2}+\mathrm{H}$

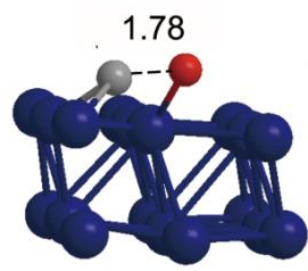

$\mathrm{C}+\mathrm{O}=\mathrm{CO}$

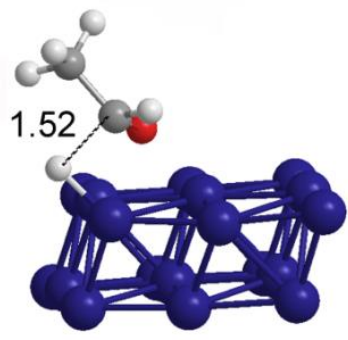

$\mathrm{CH}_{3} \mathrm{CH}_{2} \mathrm{O}=\mathrm{CH}_{3} \mathrm{CHO}+\mathrm{H}$

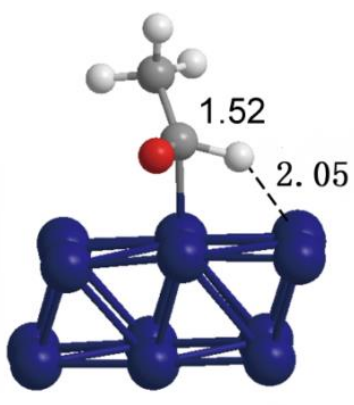

$\mathrm{CH}_{3} \mathrm{CHO}=\mathrm{CH}_{3} \mathrm{CO}+\mathrm{H}$

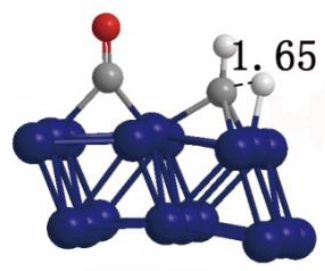

$\mathrm{CH}_{2}=\mathrm{CH}+\mathrm{H}$

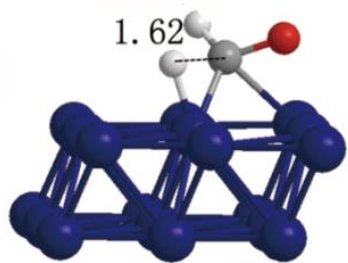

$\mathrm{H}_{2} \mathrm{CO}=\mathrm{HCO}+\mathrm{H}$

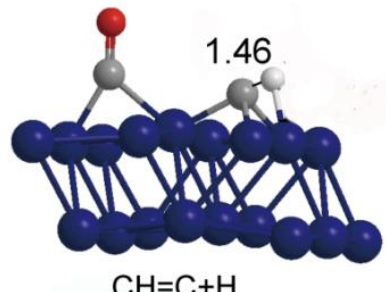

$\mathrm{CH}=\mathrm{C}+\mathrm{H}$

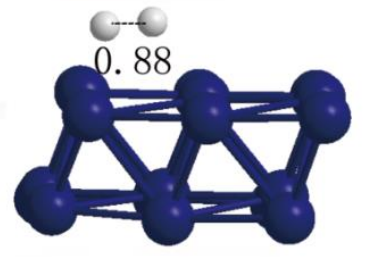

$2 \mathrm{H}=\mathrm{H}_{2}$

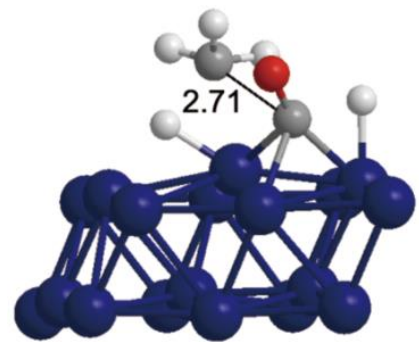

$\mathrm{CH}_{3} \mathrm{CHO}=\mathrm{CH}_{4}+\mathrm{CO}$

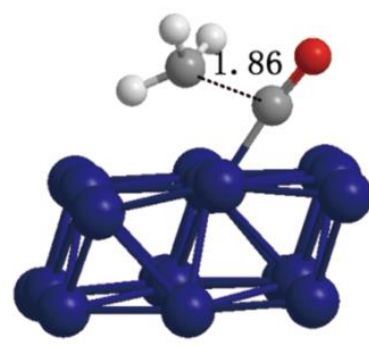

$\mathrm{CH}_{3} \mathrm{CO}=\mathrm{CH}_{3}+\mathrm{CO}$

Figure S5 The calculated TS structures for possible steps relevant to ethanol reaction on $\mathrm{Co}(0001)$ 


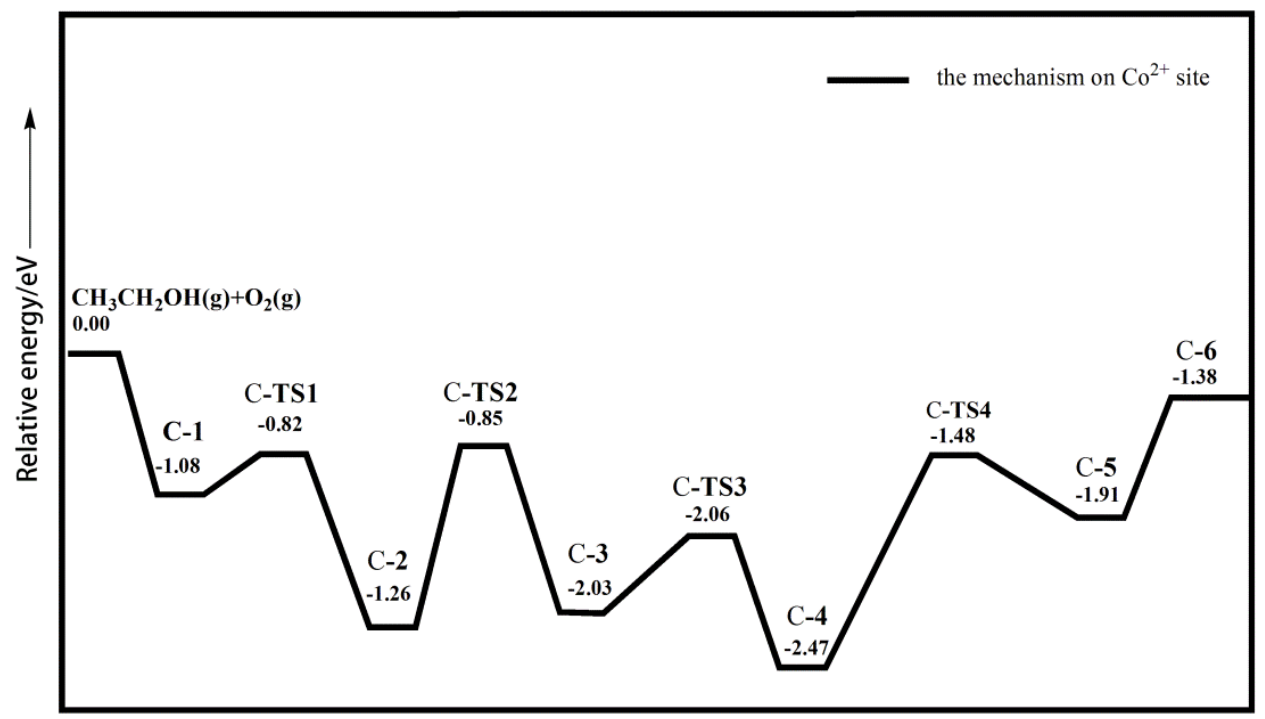

Reaction coordinates

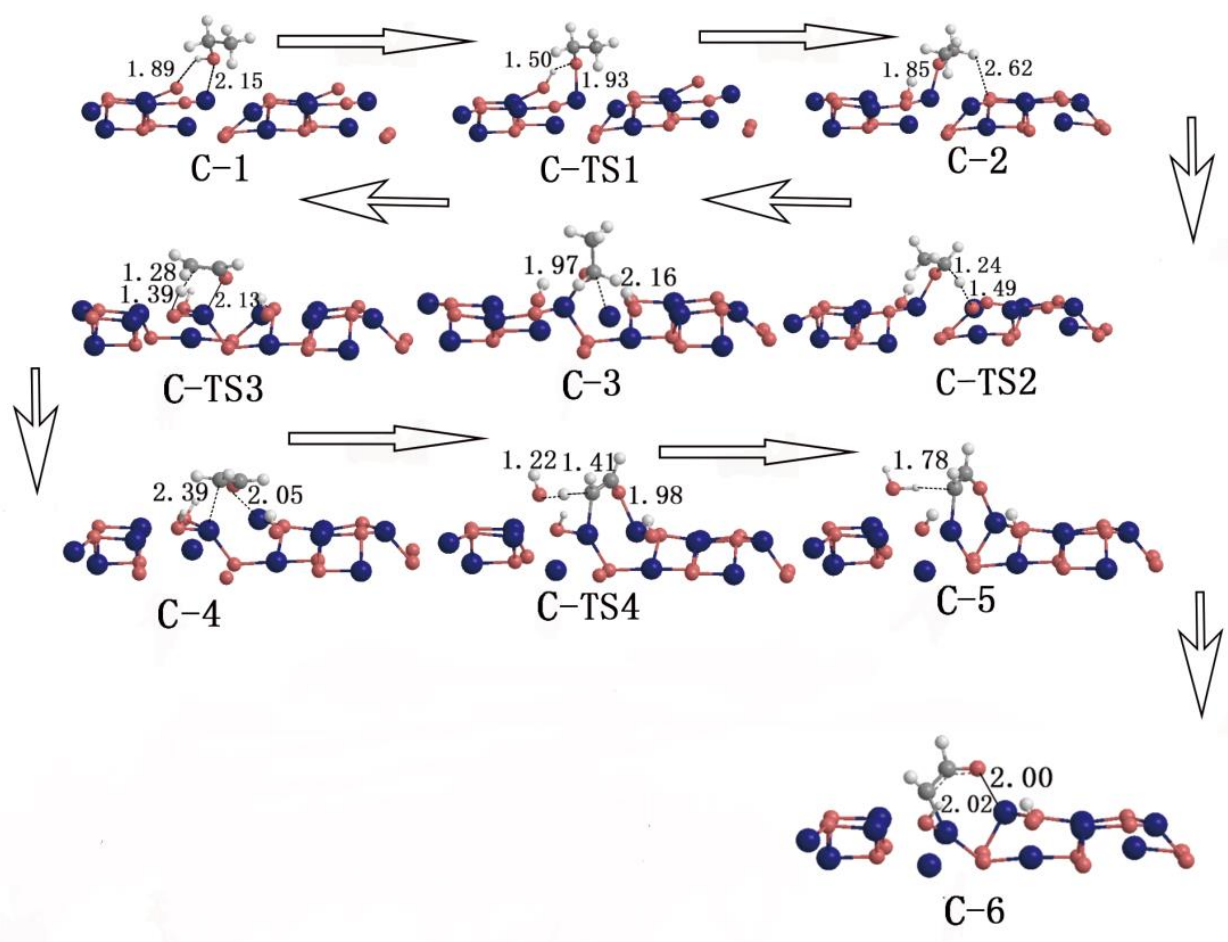

Figure S6. The reaction profiles and main configurations for the ethanol reaction mechanism on $\mathrm{Co}^{2+}$ site 


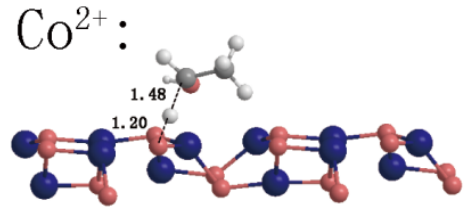

$\mathrm{CH}_{3} \mathrm{CH}_{2} \mathrm{OH}+\mathrm{O}_{3 \mathrm{f}}=\mathrm{CH}_{3} \mathrm{CHOH}+\mathrm{HO}_{3 \mathrm{f}}$

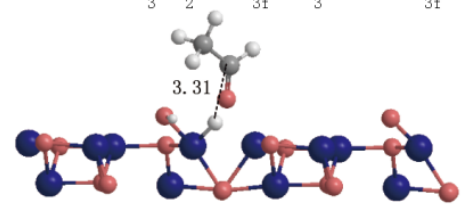

$\mathrm{CH}_{3} \mathrm{CH}_{2} \mathrm{O}+\mathrm{HO}_{3 \mathrm{f}}=\mathrm{CH}_{3} \mathrm{CHO}+\mathrm{H}_{2} \mathrm{O}_{3 \mathrm{f}}$

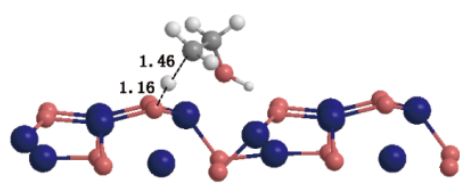

$\mathrm{CH}_{3} \mathrm{CH}_{2} \mathrm{OH}+\mathrm{O}_{3 \mathrm{f}}=\mathrm{CH}_{2} \mathrm{CH}_{2} \mathrm{OH}+\mathrm{HO}_{3 \mathrm{f}}$

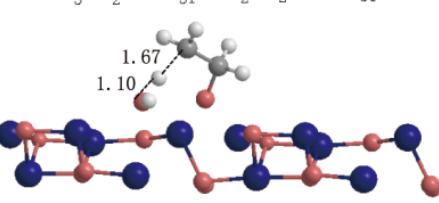

$\mathrm{CH}_{3} \mathrm{CH}_{2} \mathrm{O}+\mathrm{HO}_{3 \mathrm{f}}=\mathrm{CH}_{2} \mathrm{CH}_{2} \mathrm{OH}+\mathrm{H}_{2} \mathrm{O}_{3 \mathrm{f}}$

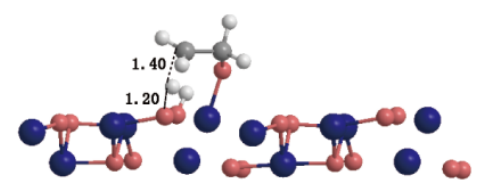

$\mathrm{CH}_{3} \mathrm{CH}_{2} \mathrm{O}+\mathrm{O}_{3 \mathrm{f}}=\mathrm{CH}_{2} \mathrm{CH}_{2} \mathrm{O}+\mathrm{HO}_{3 \mathrm{f}}$

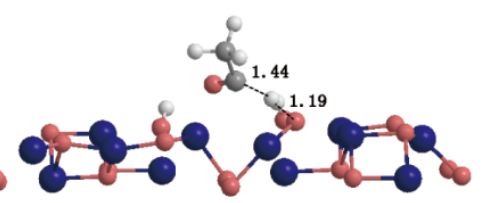

$\mathrm{CH}_{3} \mathrm{CHO}+\mathrm{O}_{3 \mathrm{f}}=\mathrm{CH}_{3} \mathrm{CO}+\mathrm{HO}_{3 \mathrm{f}}$

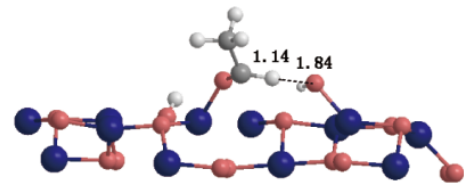

$\mathrm{CH}_{3} \mathrm{CHO}+\mathrm{HO}_{3 \mathrm{f}}=\mathrm{CH}_{3} \mathrm{CO}+\mathrm{H}_{2} \mathrm{O}_{3 \mathrm{f}}$

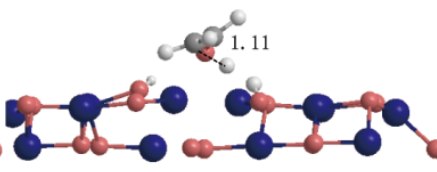

$\mathrm{CH}_{3} \mathrm{CHO}+\mathrm{HO}_{3 \mathrm{f}}=\mathrm{CH}_{2} \mathrm{CHO}+\mathrm{H}_{2} \mathrm{O}_{3 \mathrm{f}}$

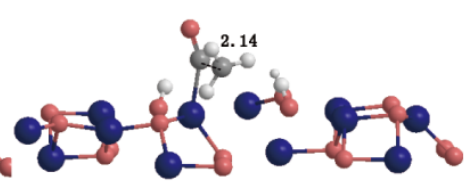

$\mathrm{CH}_{3} \mathrm{CO}=\mathrm{CH}_{3}+\mathrm{CO}$

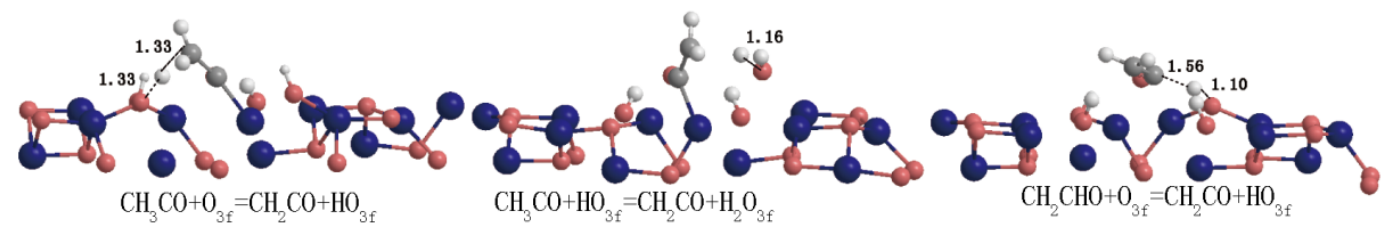

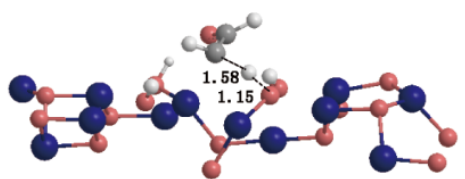

$\mathrm{CH}_{2} \mathrm{CHO}+\mathrm{O}_{3 \mathrm{f}}=\mathrm{CHCHO}+\mathrm{HO}_{3 \mathrm{f}}$

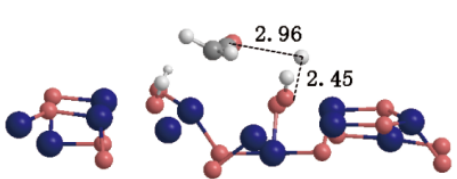

$\mathrm{CH}_{2} \mathrm{CHO}+\mathrm{HO}_{3 \mathrm{f}}=\mathrm{CH}_{2} \mathrm{CO}+\mathrm{H}_{2} \mathrm{O}_{3 \mathrm{f}}$

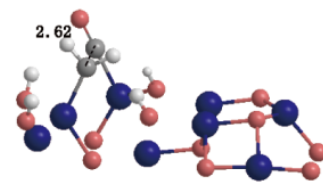

$\mathrm{CH}_{2} \mathrm{CO}=\mathrm{CH}_{2}+\mathrm{CO}$

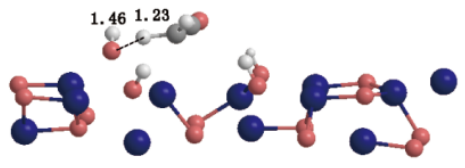

$\mathrm{CH}_{2} \mathrm{CO}+\mathrm{HO}_{3 \mathrm{f}}=\mathrm{CHCO}+\mathrm{H}_{2} \mathrm{O}_{3 \mathrm{f}}$

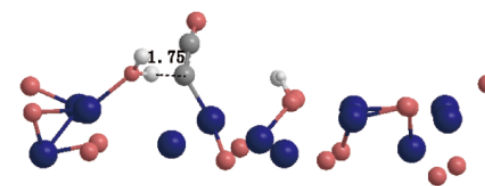

$\mathrm{CHCO}+\mathrm{HO}_{3 \mathrm{f}}=\mathrm{CCO}+\mathrm{H}_{2} \mathrm{O}_{3 \mathrm{f}}$

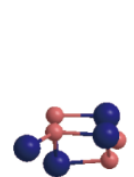

80

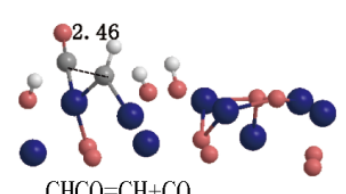

$\mathrm{CHCO}=\mathrm{CH}+\mathrm{CO}$

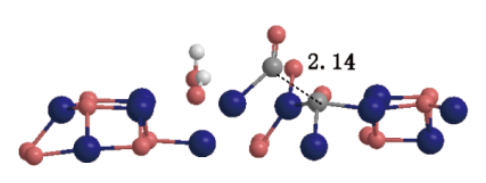

$\mathrm{CCO}=\mathrm{C}+\mathrm{CO}$

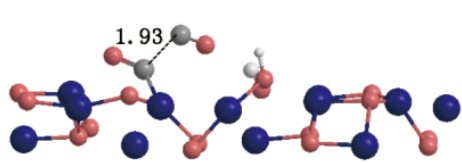

$\mathrm{COCO}=2 \mathrm{CO}$

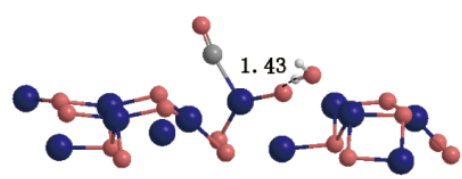

$2 \mathrm{HO}_{3 \mathrm{f}}=\mathrm{H}_{2} \mathrm{O}_{3 \mathrm{f}}+\mathrm{O}_{3 \mathrm{f}}$

Figure S7.The calculated TS structures for possible steps relevant to ethanol reaction on $\mathrm{Co}^{2+}$ site 


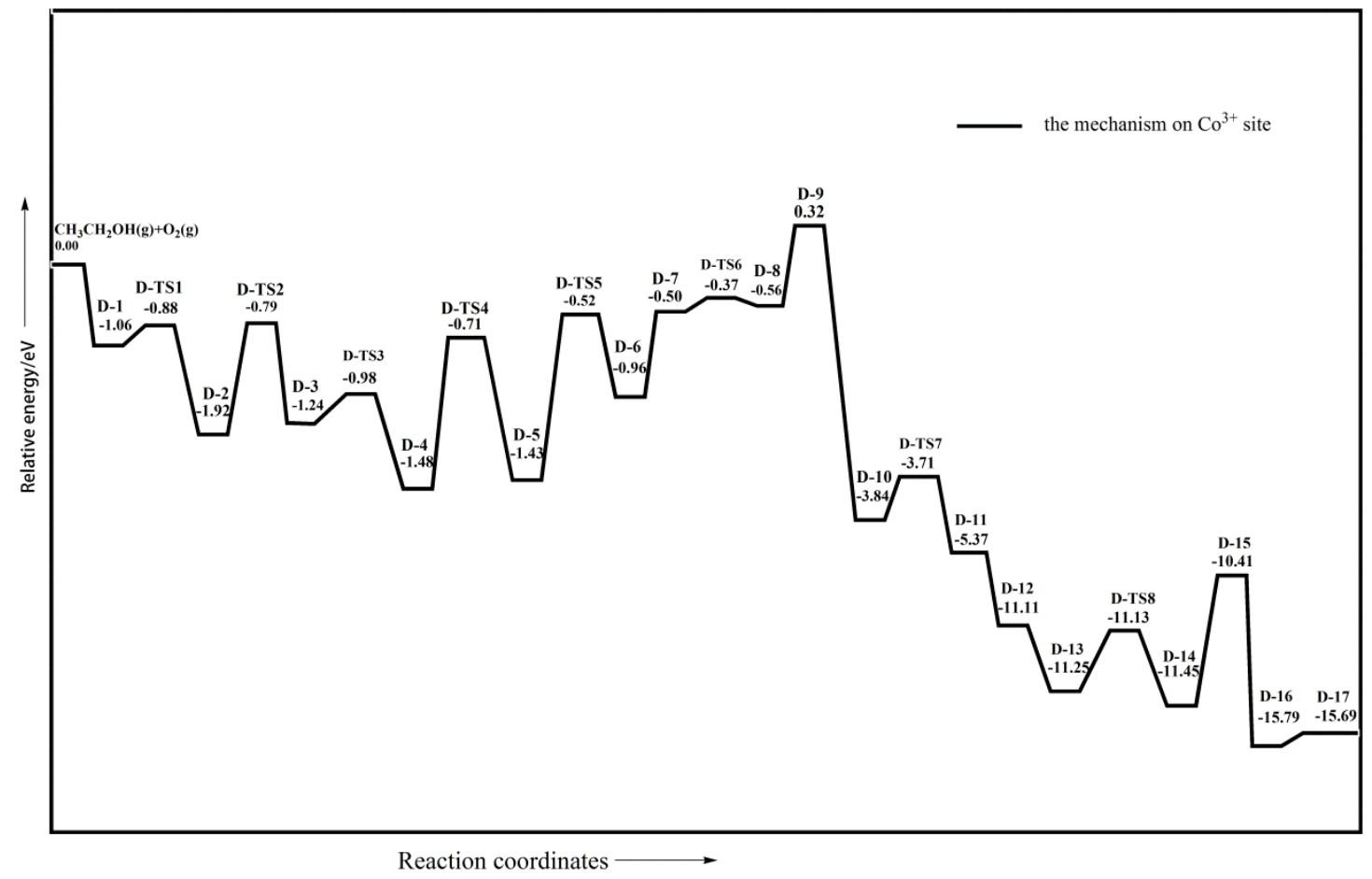



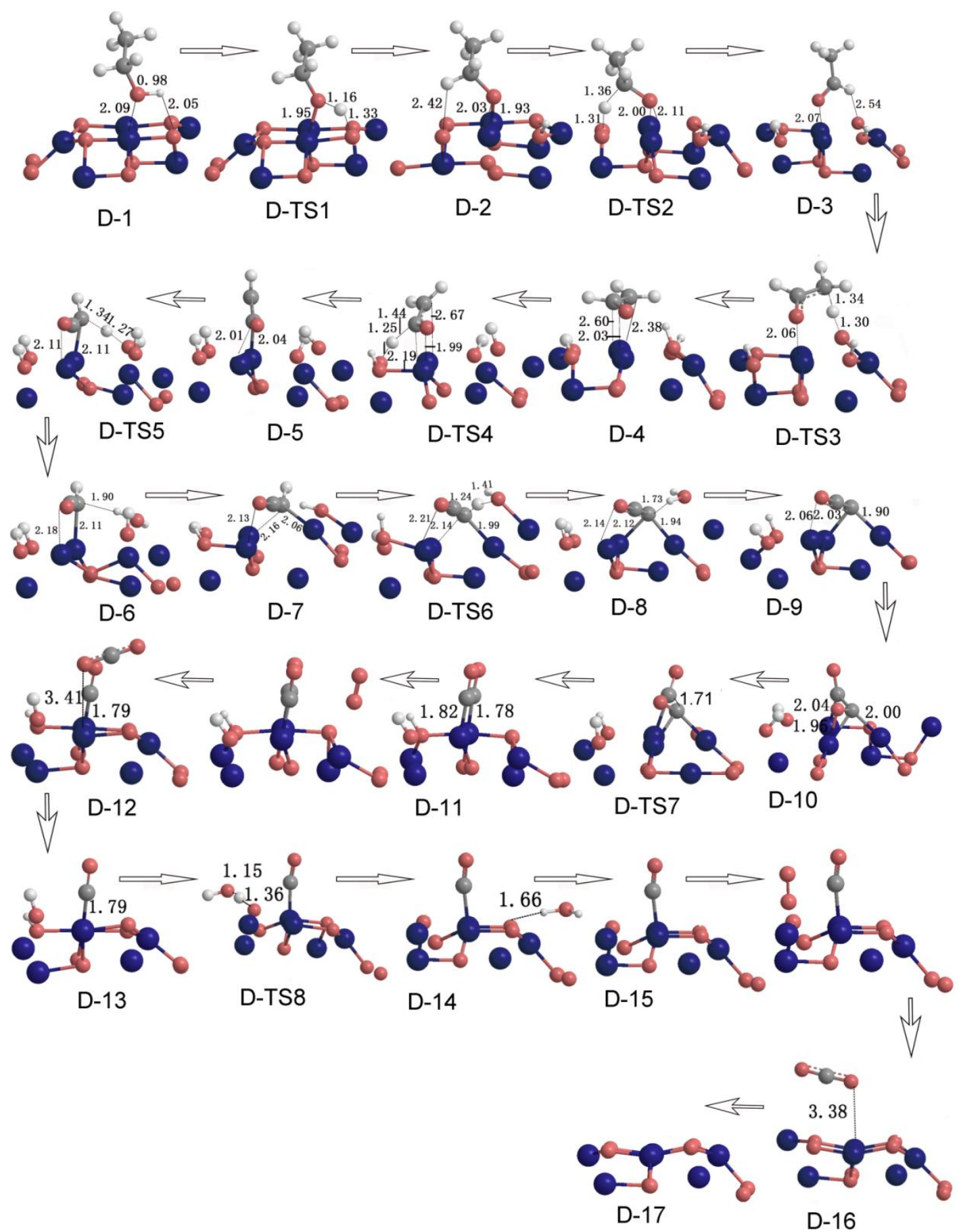

Figure S8. The reaction profiles and main configurations for the ethanol reaction mechanism on $\mathrm{Co}^{3+}$ site 
$\mathrm{Co}^{3+}$ :
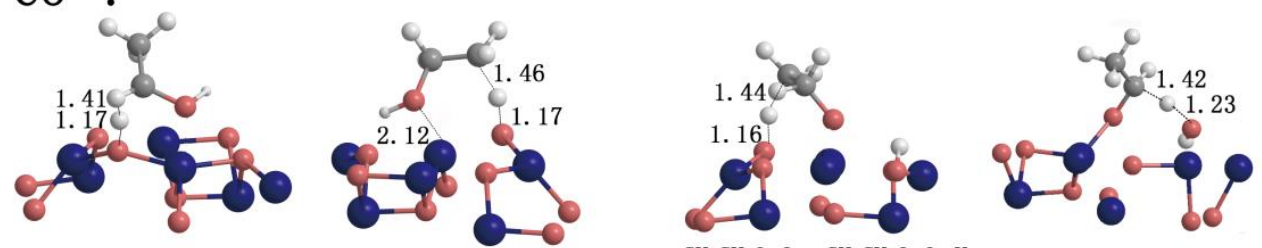

$\mathrm{CH}_{3} \mathrm{CH}_{2} \mathrm{OH}+\mathrm{O}_{3} \mathrm{f}=\mathrm{CH}_{3} \mathrm{CHOH}+\mathrm{O}_{3} \mathrm{fH} \quad \mathrm{CH}_{3} \mathrm{CH}_{2} \mathrm{OH}+\mathrm{O}_{3} \mathrm{f}=\mathrm{CH}_{2} \mathrm{CH}_{2} \mathrm{OH}+\mathrm{O}_{3} \mathrm{fH} \quad \mathrm{CH}_{3} \mathrm{CH}_{2} \mathrm{O}+\mathrm{O}_{3} \mathrm{f}=\mathrm{CH}_{2} \mathrm{CH}_{2} \mathrm{O}+\mathrm{O}_{3} \mathrm{fH}$

$\mathrm{CH}_{3} \mathrm{CH}_{2} \mathrm{O}+\mathrm{HO}_{3} \mathrm{f}=\mathrm{CH}_{3} \mathrm{CHO}+\mathrm{H}_{2} \mathrm{O}_{3} \mathrm{f}$

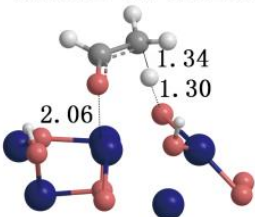

$\mathrm{CH}_{3} \mathrm{CHO}+\mathrm{O}_{3} \mathrm{f}=\mathrm{CH}_{2} \mathrm{CHO}+\mathrm{O}_{3} \mathrm{fH}$

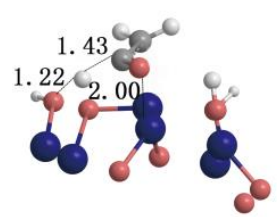

$\mathrm{CH}_{2} \mathrm{CHO}+\mathrm{H}_{3} \mathrm{f}=\mathrm{CH}_{2} \mathrm{CO}+\mathrm{H}_{2} \mathrm{O}_{3} \mathrm{f}$

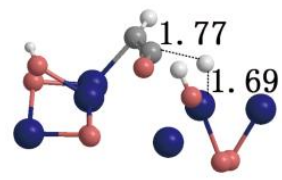

$\mathrm{CHCHO}+\mathrm{O}_{3} \mathrm{f}=\mathrm{CHCO}+\mathrm{O}_{3} \mathrm{fH}$

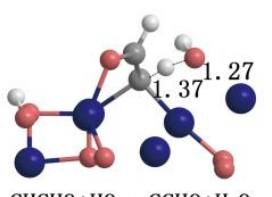

$\mathrm{CHCHO}+\mathrm{HO}_{3} \mathrm{f}=\mathrm{CCHO}+\mathrm{H}_{2} \mathrm{O}_{3} \mathrm{f}$

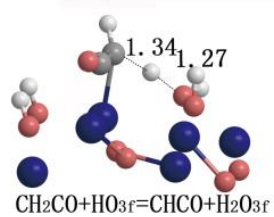

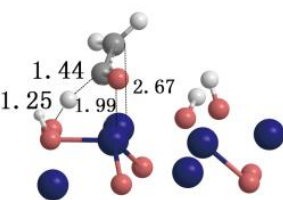

$\mathrm{CH}_{2} \mathrm{CHO}+\mathrm{O}_{3} \mathrm{f}=\mathrm{CH}_{2} \mathrm{CO}+\mathrm{O}_{3} \mathrm{fH}$

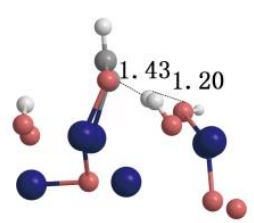

$\mathrm{CH}_{2} \mathrm{CHO}+\mathrm{H}_{3} \mathrm{f}=\mathrm{CHCHO}+\mathrm{H}_{2} \mathrm{O}_{3} \mathrm{f}$
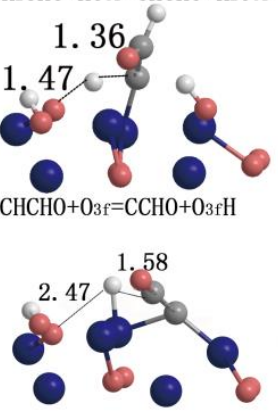

$\mathrm{CCHO}+03 \mathrm{f}=\mathrm{CCO}+03 \mathrm{fH}(\mathrm{TS} 1)$
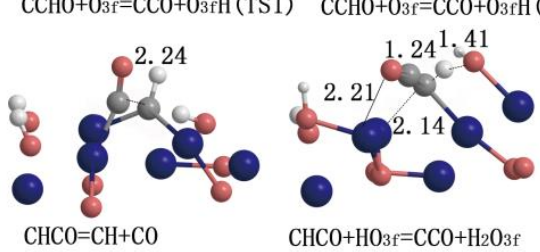

$\mathrm{CHCO}+\mathrm{HO}_{3} \mathrm{f}=\mathrm{CCO}+\mathrm{H}_{2} \mathrm{O}_{3} \mathrm{f}$
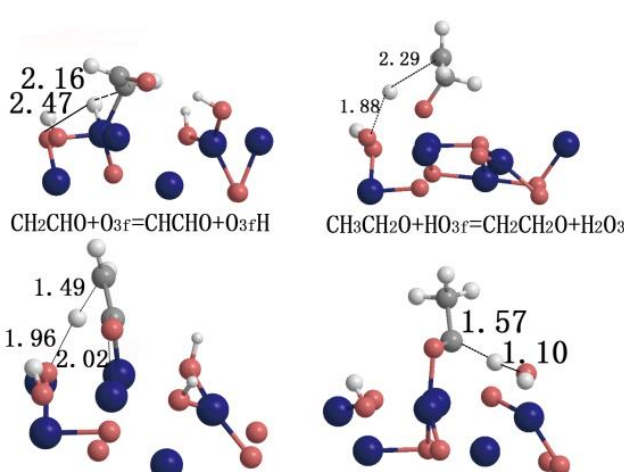

$\mathrm{CH}_{3} \mathrm{CO}+\mathrm{O}_{3} \mathrm{f}=\mathrm{CH}_{2} \mathrm{CO}+\mathrm{O}_{3} \mathrm{fH}$

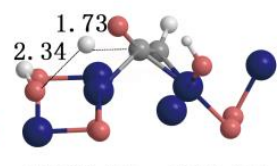

$\mathrm{CHCHO}+\mathrm{HO}_{3} \mathrm{f}=\mathrm{CHCO}+\mathrm{H}_{2} \mathrm{O}_{3} \mathrm{f}$
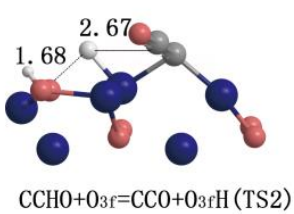

$\mathrm{CH}_{2} \mathrm{CO}=\mathrm{CO}+\mathrm{CH}_{2}$

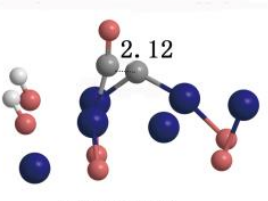

$\mathrm{CCO}=\mathrm{C}+\mathrm{CO}$

Figure S9. The calculated TS structures for possible steps relevant to ethanol reaction on $\mathrm{Co}^{3+}$ site 